\title{
Molecular Salts with Diquat-based Electron Acceptors for Nonlinear Optics
}

Benjamin J. Coe, James A. Harris, Bruce S. Brunschwig, Javier Garín and Jesús Orduna

\section{Supporting Information}

\section{Complete Reference 6}

(6) (a) Coe, B. J.; Harris, J. A.; Asselberghs, I.; Clays, K.; Olbrechts, G.; Persoons, A.; Hupp, J. T.; Johnson, R. C.; Coles, S. J.; Hursthouse, M. B.; Nakatani, K. Adv. Funct. Mater. 2002, 12, 110-116. (b) Coe, B. J.; Harris, J. A.; Asselberghs, I.; Wostyn, K.; Clays, K.; Persoons, A.; Brunschwig, B. S.; Coles, S. J.; Gelbrich, T.; Light, M. E.; Hursthouse, M. B.; Nakatani, K. Adv. Funct. Mater. 2003, 13, 347-357.

\section{Experimental Details}

Physical Measurements. ${ }^{1} \mathrm{H}$ NMR spectra were recorded on a Varian Gemini 200 spectrometer and all shifts are referenced to TMS. The fine splitting of pyridyl or phenyl ring patterns is ignored and the signals are reported as simple doublets, with $J$ values referring to the two most intense peaks. Elemental analyses were performed by the Microanalytical Laboratory, University of Manchester and UV-visible spectra were obtained by using a Hewlett Packard 8452A diode array spectrophotometer. Cyclic voltammetric measurements were carried out by using an EG\&G PAR model 283 potentiostat/galvanostat. A single-compartment cell was used with a $\mathrm{Ag}-\mathrm{AgCl}$ reference electrode separated by a salt bridge from the platinum-disc working electrode and platinum-wire auxiliary electrode. Anhydrous acetonitrile (HPLC grade, distilled under argon from $\mathrm{CaH}_{2}$ ) was used as the solvent and tetra- $n$-butyl ammonium hexafluorophosphate, twice recrystallized from ethanol and dried in vacuo, was used as supporting electrolyte. Solutions containing ca. $10^{-3} \mathrm{M}$ analyte $(0.1 \mathrm{M}$ electrolyte) were deaerated by purging with $\mathrm{N}_{2}$. All $E_{1 / 2}$ values were calculated from $\left(E_{\mathrm{pa}}+E_{\mathrm{pc}}\right) / 2$ at a scan rate of $200 \mathrm{mV} \mathrm{s}^{-1}$.

Data for 2-Methyl-11-(E-4-(dimethylamino)phenylethenyl)-6,7-dihydrodipyrido[1,2-a:2',1'-c]pyrazinediium hexafluorophosphate (2). $\delta_{\mathrm{H}}\left(\mathrm{CD}_{3} \mathrm{CN}\right)$ 8.70-8.69 $\left(2 \mathrm{H}, \mathrm{m}, \mathrm{C}_{5} \mathrm{H}_{3} \mathrm{~N}\right), 8.58\left(1 \mathrm{H}, \mathrm{s}, \mathrm{C}_{5} \mathrm{H}_{3} \mathrm{~N}\right), 8.44\left(1 \mathrm{H}, \mathrm{d}, J=7.0 \mathrm{~Hz}, \mathrm{C}_{5} \mathrm{H}_{3} \mathrm{~N}\right), 8.09-8.04(2 \mathrm{H}, \mathrm{m}$, $\mathrm{C}_{5} \mathrm{H}_{3} \mathrm{~N}$ and $\left.\mathrm{CH}\right), 7.92\left(1 \mathrm{H}, \mathrm{d}, J=8.1 \mathrm{~Hz}, \mathrm{C}_{5} \mathrm{H}_{3} \mathrm{~N}\right), 7.68\left(2 \mathrm{H}, \mathrm{d}, J=8.7 \mathrm{~Hz}, \mathrm{C}_{6} \mathrm{H}_{4}\right), 7.16(1 \mathrm{H}$, $\mathrm{d}, J=15.6 \mathrm{~Hz}, \mathrm{CH}), 6.85\left(2 \mathrm{H}, \mathrm{d}, J=8.8 \mathrm{~Hz}, \mathrm{C}_{6} \mathrm{H}_{4}\right), 5.00-4.94\left(2 \mathrm{H}, \mathrm{m}, \mathrm{CH}_{2}\right), 4.86-4.81(2 \mathrm{H}$, m, $\left.\mathrm{CH}_{2}\right), 3.11\left(6 \mathrm{H}, \mathrm{s}, \mathrm{NMe}_{2}\right), 2.82(3 \mathrm{H}, \mathrm{s}, \mathrm{Me})$. Anal. Calcd (\%) for $\mathrm{C}_{23} \mathrm{H}_{25} \mathrm{~F}_{12} \mathrm{~N}_{3} \mathrm{P}_{2} \bullet \mathrm{H}_{2} \mathrm{O}: \mathrm{C}$, 42.41; H, 4.18; N, 6.45. Found: C, 42.68; H, 3.90; N, 6.42. 
Data for 2,11-Bis $(E-4-($ dimethylamino $)$ phenylethenyl)-6,7-dihydrodipyrido[1,2-a:2',1'-c]pyrazinediium hexafluorophosphate $(3) . \delta_{\mathrm{H}}\left(\mathrm{CD}_{3} \mathrm{CN}\right) 8.64(2 \mathrm{H}$, $\left.\mathrm{s}, \mathrm{C}_{5} \mathrm{H}_{3} \mathrm{~N}\right), 8.42\left(2 \mathrm{H}, \mathrm{d}, J=6.7 \mathrm{~Hz}, \mathrm{C}_{5} \mathrm{H}_{3} \mathrm{~N}\right), 8.07(2 \mathrm{H}, \mathrm{d}, J=15.8 \mathrm{~Hz}, \mathrm{CH}), 7.92(2 \mathrm{H}, \mathrm{d}, J=$ $\left.6.4 \mathrm{~Hz}, \mathrm{C}_{5} \mathrm{H}_{3} \mathrm{~N}\right), 7.81\left(4 \mathrm{H}, \mathrm{d}, J=9.0 \mathrm{~Hz}, \mathrm{C}_{6} \mathrm{H}_{4}\right), 7.18(2 \mathrm{H}, \mathrm{d}, J=15.3 \mathrm{~Hz}, \mathrm{CH}), 6.86(4 \mathrm{H}, \mathrm{d}, J$ $\left.=8.5 \mathrm{~Hz}, \mathrm{C}_{6} \mathrm{H}_{4}\right), 4.81\left(4 \mathrm{H}, \mathrm{s}, \mathrm{CH}_{2}\right), 3.11\left(12 \mathrm{H}, \mathrm{s}, \mathrm{NMe}_{2}\right)$. Anal. Calcd (\%) for $\mathrm{C}_{32} \mathrm{H}_{34} \mathrm{~F}_{12} \mathrm{~N}_{4} \mathrm{P}_{2} \bullet 1.5 \mathrm{H}_{2} \mathrm{O}$ : C, 48.55; H, 4.71; N, 7.08. Found: C, 48.33; H, 4.57; N, 6.92.

Stark Spectroscopy. The apparatus, experimental methods and data analysis procedure were exactly as previously reported. ${ }^{1-3}$ Representative absorption and electroabsorption spectra for salts $\mathbf{2}$ and $\mathbf{3}$ are shown in Figure S1.
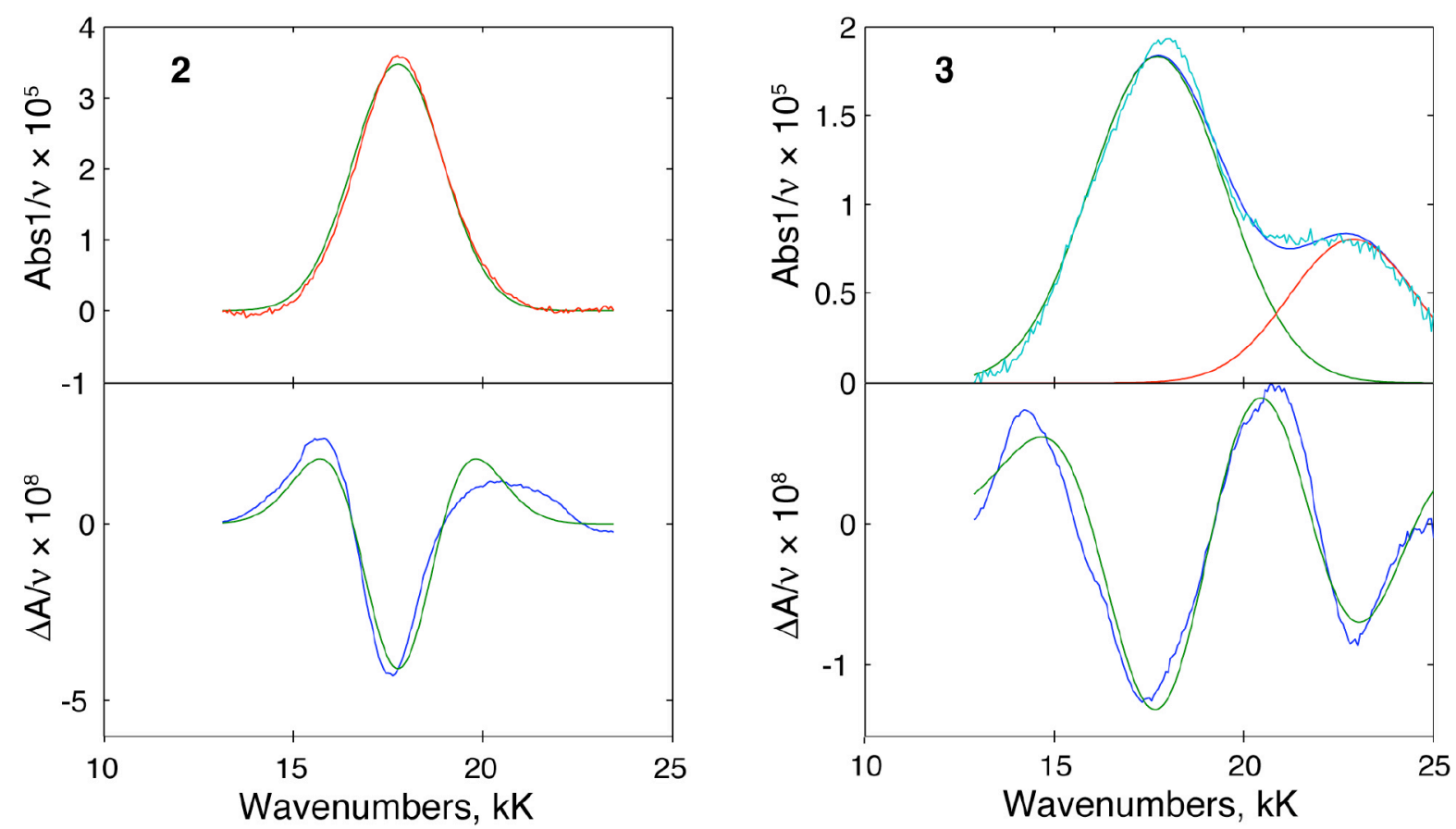

Figure S1. Stark spectra and calculated fits for $\mathbf{2}$ and $\mathbf{3}$ in external electric fields of 2.77 and $2.76 \times 10^{7} \mathrm{~V} \mathrm{~m}^{-1}$, respectively. Top panel: absorption spectrum showing experimental data (red) and fit (green) for $\mathbf{2}$ and two Gaussian curves (red and green) utilized in data fitting for 3; bottom panel: electroabsorption spectrum, experimental (blue) and fits (green) according to the Liptay equation. ${ }^{4}$

Computational Procedures. All theoretical calculations were performed on the isolated gas-phase cations by using the Gaussian $03^{5}$ program. No attempts were made to include solvents, which may explain some of the apparent differences between the experimental observations and theory. The molecular geometries were optimized using the B3P86 ${ }^{6}$ functional and the LanL2DZ $\mathrm{DZ}^{7}$ basis set, assuming $C_{s}$ symmetry for the cation in salt $\mathbf{1}$ and $C_{2}$ symmetry for the cation in $\mathbf{3}$. The same model chemistry was used for TD-DFT calculations and the excited state dipole moments were calculated by using the one particle RhoCI density. 
Molecular hyperpolarizabilities at zero frequency were calculated by either the finite field approach using the B3P86/LanL2DZ model or the coupled perturbed Hartree Fock method (CPHF) using the HF/LanL2DZ model. The default Gaussian 03 parameters were used in every case. Molecular orbital contours were plotted using Molekel $4.3,{ }^{8}$ as shown in Figure S2 for the cationic chromophore in salt 3 .
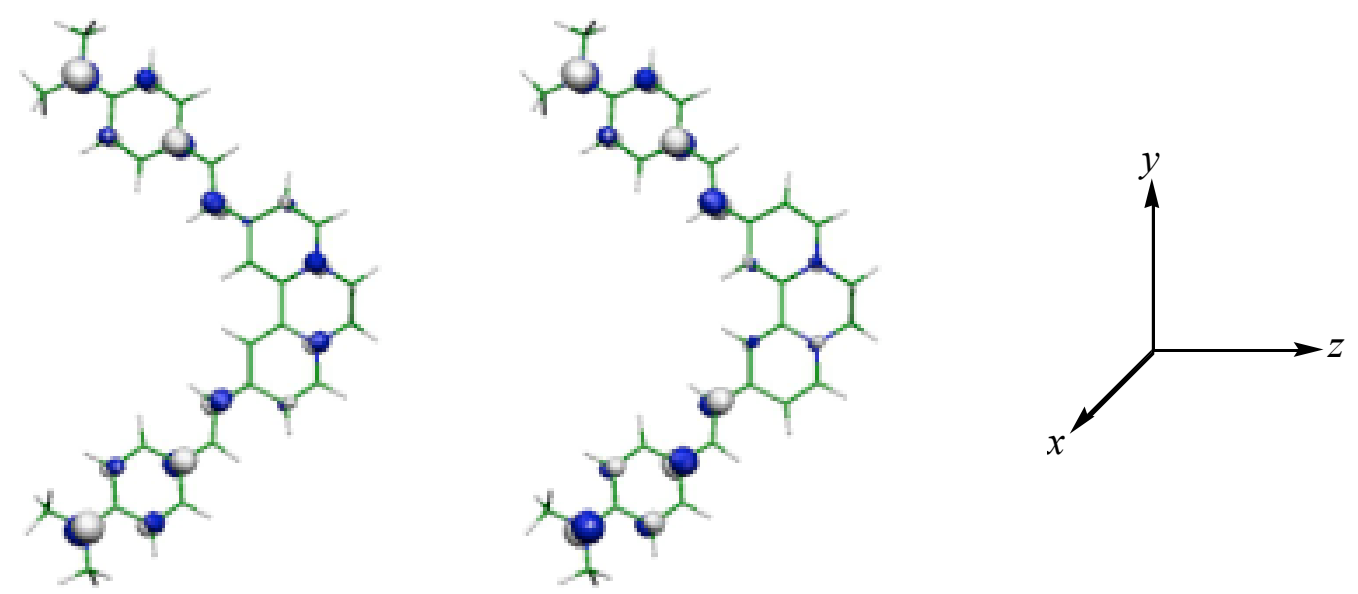

HOMO $-1 b-10.37 \mathrm{eV}$
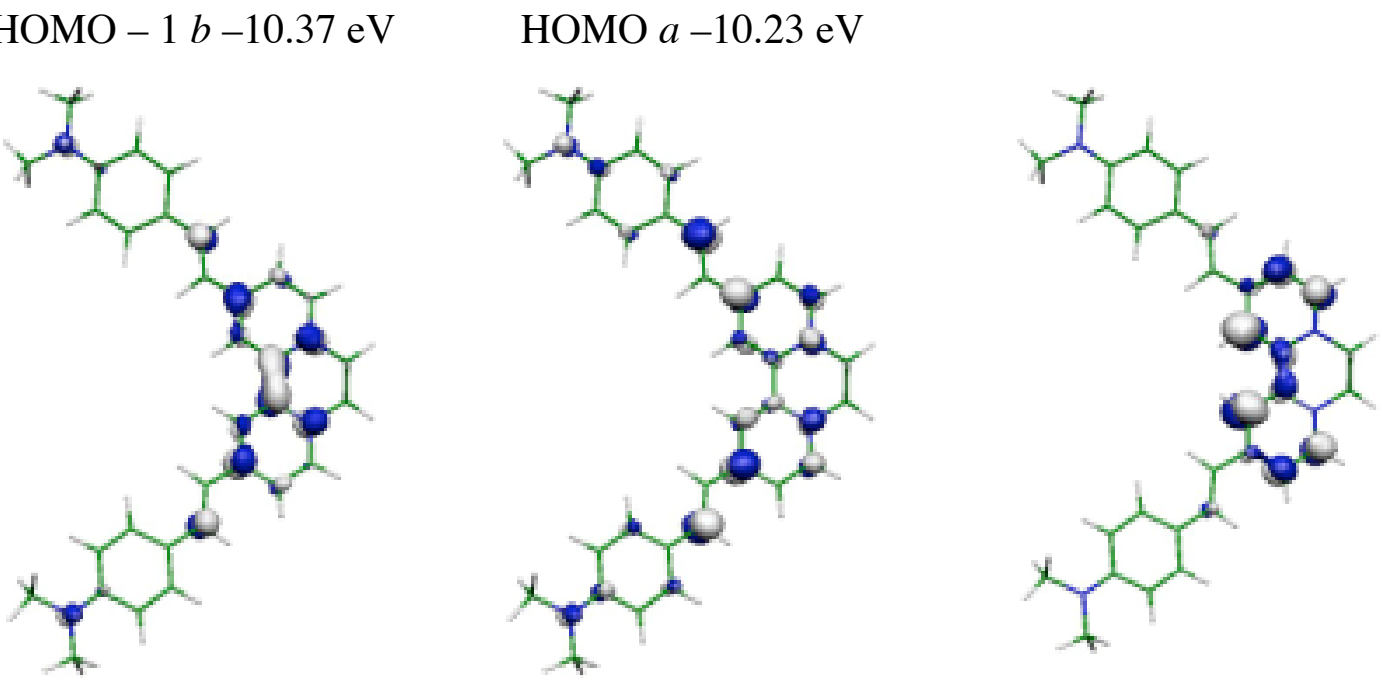

LUMO $b-8.44 \mathrm{eV}$

$$
\mathrm{LUMO}+1 a-7.66 \mathrm{eV}
$$$$
\mathrm{LUMO}+2 b-7.13 \mathrm{eV}
$$

Figure S2. 0.05 contour plots of the MOs involved in the lowest energy transitions of the cation in salt $\mathbf{3}$, calculated from TD-DFT.

(1) Shin, Y. K.; Brunschwig, B. S.; Creutz, C.; Sutin, N. J. Phys. Chem. 1996, 100, 8157-8169.

(2) Coe, B. J.; Harris, J. A.; Brunschwig, B. S. J. Phys. Chem. A 2002, 106, 897-905.

(3) Coe, B. J.; Harris, J. A.; Brunschwig, B. S.; Garín, J.; Orduna, J.; Coles, S. J.; Hursthouse, M. B. J. Am. Chem. Soc. 2004, 126,10418-10427.

(4) Liptay, W. In Excited States, Vol. 1; Lim, E. C., Ed.; Academic Press, New York, 1974, 
pp. 129-229.

(5) Gaussian 03, Revision B.05, Frisch, M. J.; Trucks, G. W.; Schlegel, H. B.; Scuseria, G. E.; Robb, M. A.; Cheeseman, V. G.; Montgomery, J. A., Vreven, Jr. T.; Kudin, K. N.; Burant, J. C.; Millam, J. M.; Iyengar, S. S.; Tomasi, J.; Barone, V.; Mennucci, B.; Cossi, M.; Scalmani, G.; Rega, N.; Petersson, G. A.; Nakatsuji, H.; Hada, M.; Ehara, M.; Toyota, K.; Fukuda, R.; Hasegawa, J.; Ishida, M.; Nakajima, T.; Honda, Y.; Kitao, O.; Nakai, H.; Klene, M.; Li, X.; Knox, J. E.; Hratchian, H. P.; Cross, J. B.; Adamo, C.; Jaramillo, J.; Gomperts, R.; Stratmann, R. E.; Yazyev, O.; Austin, A. J.; Cammi, R.; Pomelli, C.; Ochterski, J. W.; Ayala, P. Y.; Morokuma, K.; Voth, G. A.; Salvador, P.; Dannenberg, J. J.; Zakrzewski, V. G.; Dapprich, S.; Daniels, A. D.; Strain, M. C.; Farkas, O.; Malick, D. K.; Rabuck, A. D.; Raghavachari, K.; Foresman, J. B.; Ortiz, J. V.; Cui, Q.; Baboul, A. G.; Clifford, S.; Cioslowski, J.; Stefanov, B. B.; Liu, G.; Liashenko, A.; Piskorz, P.; Komaromi, I.; Martin, R. L.; Fox, D. J.; Keith, T.; Al-Laham, M. A.; Peng, C. Y.; Nanayakkara, A.; Challacombe, M.; Gill, P. M. W.; Johnson, B.; Chen, W.; Wong, M. W.; Gonzalez, C.; Pople, J. A.; Gaussian, Inc., Pittsburgh PA, 2003.

(6) The B3P86 Functional consists of Becke's three parameter hybrid functional (Becke, A. D. J. Chem. Phys. 1993, 98, 5648-5652) with the nonlocal correlation provided by the Perdew 86 expression: Perdew, J. P. Phys. Rev. B 1986, 33, 8822-8824.

(7) D95 on first row: Dunning, T. H.; Hay P. J., in Modern Theoretical Chemistry: Schaefer, H. F. III, Ed.; Plenum: New York, 1976, vol. 3, 1; Los Alamos ECP plus DZ on Na-Bi: (a) Hay, P. J.; Wadt, W. R. J. Chem. Phys. 1985, 82, 270-283. (b) Wadt, W. R.; Hay, P. J. J. Chem. Phys. 1985, 82, 284-298. (c) Hay, P. J.; Wadt, W. R. J. Chem. Phys. 1985, 82, 299-310.

(8) Portmann, S.; Lüthi, H. P. Chimia 2000, 54, 766-770. 